\title{
The infinite word problem and limit sets in Fuchsian groups
}

\author{
CAROLINE SERIES \\ Mathematics Institute, University of Warwick, Coventry CV4 7AL, England
}

(Received 15 November 1980 and revised 20 March 1981)

Abstract. Let $\Gamma$ be a finitely generated non-elementary Fuchsian group acting in the disk. With the exception of a small number of co-compact $\Gamma$, we give a representation of $g \in \Gamma$ as a product of a fixed set of generators $\Gamma_{0}$ in a unique shortest 'admissible form'. Words in this form satisfy rules which after a suitable coding are of finite type. The space of infinite sequences $\Sigma$ of generators satisfying the same rules is identified in a natural way with the limit set $\Lambda$ of $\Gamma$ by a map which is bijective except at a countable number of points where it is two to one. We use the theory of Gibbs measures on $\Sigma$ to construct the so-called Patterson measure on $\Lambda$ [8], [9]. This measure is, in fact, Hausdorff $\delta$-dimensional measure on $\Lambda$, where $\delta$ is the exponent of convergence of $\Gamma$.

\section{Introduction}

Let us begin with a picture and an example. Suppose $\Gamma$ is the free group on two generators $a$ and $b$. We may obtain a representation of $\Gamma$ as a Fuchsian group acting in the unit disk $D$ as follows: take any four disjoint circular $\operatorname{arcs} C_{a}, C_{a^{-1}}$, $C_{b}, C_{b^{-1}}$, orthogonal to the unit circle $S^{1}$, and let $a, b$ be the linear fractional transformations which map the exterior of $C_{a}$ onto the interior of $C_{a}^{-1}$, the exterior of $C_{b}$ onto the interior of $C_{b^{-1}}$, and map $S^{1}$ to itself (see figure 1). Then the group generated by $a, b$ is $\Gamma$ and $\Gamma$ has fundamental region $R$, the region outside all four circles.

Now every element of $\Gamma$ has a unique representation as a reduced word in the generators $\Gamma_{0}=\left\{a, b, a^{-1}, b^{-1}\right\}$ : namely, a word in which an element of $\Gamma_{0}$ is never followed by its inverse. Such words can be thought of as paths in the graph $G(\Gamma)$ of $\Gamma$. For consider the orbit $\Gamma 0$ of $0 \in D$ (we assume $0 \in R$ ). Join the vertices $g 0$, $g^{\prime} 0$ if and only if $g^{-1} g^{\prime} \in \Gamma_{0}$, and label the directed edge from $g 0$ to $g^{\prime} 0$ by $g^{-1} g^{\prime}$. Now if $g \in \Gamma$ there is a unique path in the graph from 0 to $g 0$ and this gives exactly the representation of $g$ as a reduced word.

It is clear geometrically that the ends of $G(\Gamma)$ are precisely the limit set $\Lambda$ of $\Gamma$, and one can define a bijection $\Sigma \rightarrow \Lambda$ where $\Sigma$ is the space of infinite reduced words in $\Gamma_{0}$. This map extends the natural embedding $\Gamma \rightarrow \Gamma 0$ of $\Gamma$ into $D$. The shift $\sigma$ on $\Sigma$ induces a map $f: \Lambda \rightarrow \Lambda$. $f$ is described more transparently as follows: if $x \in \Lambda$ then $f(x)=e x$ if $x \in C_{e^{-1}}, e \in \Gamma_{0}$. By construction $f$ is conjugate to a Markov shift of finite type. One also sees that $x, y \in \Lambda, x=g y$ if and only if $f^{n} x=f^{m} y$ for some 


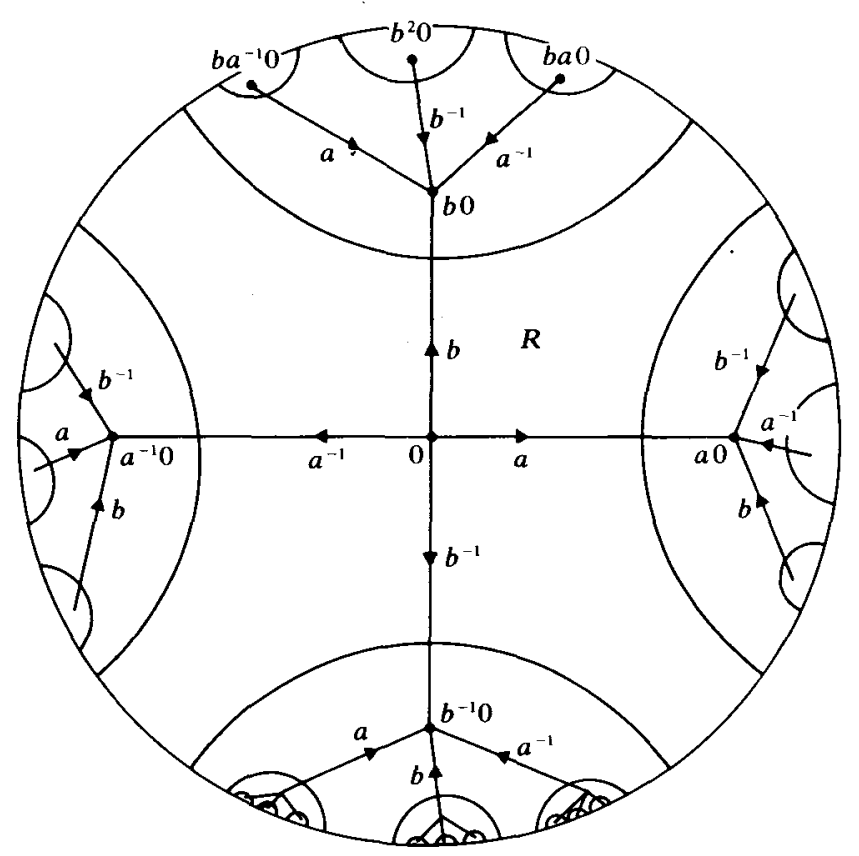

FIGURE 1

$n, m$; in other words $\Gamma$ and $f$ are orbit equivalent on $\Lambda$. If $C_{e}$ is chosen to be within the isometric circle of $e$, then $f$ is expanding; more generally, one shows $f^{N}$ is always expanding for some $N$ (see $\S 5$ ).

\section{Generalization}

One would like an analogous construction for any finitely generated Fuchsian group $\Gamma$; this we make in the present paper. The main difficulty is, given a fixed set of generators $\Gamma_{0}$ of $\Gamma$, to describe a canonical representation of elements of $\Gamma$ as words in $\Gamma_{0}$. In addition, one wants the rules governing this representation to be of finite type. For the free group this was trivial because the only relations were $e e^{-1}=1$, $e \in \Gamma_{0}$, In the general case we need an explicit solution of the word problem in $\Gamma$ : This is essentially the work of Dehn [4].

Using Dehn's methods we show that, except for a small number of co-compact groups $\Gamma$ (see theorem 2.7 for a precise statement), it is posible to specify a certain canonical representation of each $g \in \Gamma$ as a shortest word in the generators $\Gamma_{0}$. Under a suitable coding the rules governing this representation are of finite type.

The map $\Gamma \rightarrow \Gamma 0$ extends to a map $\pi: \Sigma \rightarrow \Lambda$ where $\Sigma$ is the space of infinite words in $\Gamma_{0}$ in canonical form and $\Lambda$ is the limit set of $\Gamma . \pi$ is surjective and injective except at a countable set of points where it is two to one. The shift $\sigma$ on $\Sigma$ induces a map $f: \Lambda \rightarrow \Lambda$ which is orbit equivalent to $\Gamma$ on $\Lambda$.

In [3] we constructed such maps $f$ for groups $\Gamma$ where $D / \Gamma$ had finite area. For example, when $\Gamma=\mathrm{SL}(2, \mathbb{Z}), f$ was essentially the continued fraction transformation. However, we made a direct construction only for groups with very special 
fundamental regions; to pass to the general case we applied the theory of quasiconformal maps. Here the maps $f$ are the same but the construction is quite different and general; in fact for groups $\Gamma, \Gamma^{\prime}$ with the same abstract generators and relations the spaces of infinite words $\Sigma_{\Gamma}, \Sigma_{\Gamma^{\prime}}$ are identical and the induced map $\Lambda_{\Gamma} \rightarrow \Lambda_{\Gamma^{\prime}}$ exhibits a quasi-conformal deformation of the limit sets. It is amusing to note that Dehn's solution of the word problem (1912) is essentially the same as the $f$ expansion rules derived in [3].

In the last section we restrict attention to groups $\Gamma$ with no parabolic elements. We show that there exists $N \in \mathbb{N}$ so that $f^{N}$ is uniformly expanding. By applying the theory of Gibbs measures [1] to $\Sigma$ we show that there is a probability measure $\mu$ on $\Lambda$ and $0<\delta \leq 1$ so that:

(1) If $\phi(x)=-\log \left|f^{\prime}(x)\right|, x \in \Lambda$, then $P(\delta \phi)$, the pressure of $\delta \phi$, is zero.

(2) The measure $\nu$ which is a fixed point for the Perron-Frobenius operator of $\phi($ see $[1])$ satisfies

$$
\frac{d g_{*} \nu(x)}{d \nu}=\left|g^{\prime}(x)\right|^{\delta}, \quad x \in S^{1}
$$

$\nu$ is Hausdorff $\delta$-dimensional measure on $\Lambda$ and is also the Patterson measure constructed in [8] and [9].

(3) $\mu$ is equivalent to $\nu$ and is invariant and ergodic for $f$, hence ergodic for $\Gamma$.

(4) $\delta=1$ if and only if $\Lambda=S^{1}$.

(5) The Poincaré series $\sum_{g \in \Gamma} \exp (-s H(0, g 0))$, where $H$ is hyperbolic distance, has exponent of convergence $\delta$ and diverges at $s=\delta$.

None of the results of the last section are new except the method of construction of $\mu$ (and of course the invariance of $\mu$ with respect to $f$ ). Otherwise everything is contained in [8] and [9]. We should also mention the work of Floyd [5] in which a map similar to our $\pi$ is constructed from an abstract completion of the space of finite words in $\Gamma_{0}$ onto $\Lambda$.

The author would like to thank Paddy Patterson for conversations which gave birth to the idea of a connection between his measures and Gibbs states, and Dennis Sullivan for ideas and encouragements too numerous to mention.

\section{Preliminaries}

Let us recall briefly the relevant facts about Fuchsian groups acting in the unit disk $D=\{z \in \mathbb{C}:|z|<1\}$. Such a group is by definition a discrete subgroup of the group

$$
\left\{\left(\begin{array}{ll}
a & b \\
b & a
\end{array}\right): a, b \in \mathbb{C},|a|^{2}-|b|^{2}=1\right\}
$$

of conformal automorphisms of the disk. Its limit set $\Lambda$, the set of accumulation points of orbits, is a subset of the unit circle $S^{1}=\{z:|z|=1\}$. Elements of $\Gamma$ are called parabolic, hyperbolic or elliptic according as they have one or two fixed points on $S^{1}$, or one fixed point in $D$. We shall always make the assumption that $\Gamma$ is finitely generated.

With the metric $d s=2|d z| /\left(1-|z|^{2}\right), D$ becomes a model for non-Euclidean geometry in which the straight lines are circular arcs orthogonal to $S^{1}$. By a 
fundamental region for $\Gamma$ we mean a geodesically convex polygon $R \subseteq \bar{D}$ with a finite number of sides (possibly including arcs of $S^{1}$ ), such that no two interior points of $R$ are conjugate under $\Gamma$ and every point in $D$ is conjugate to a point in $\bar{R}$. Many such fundamental regions exist for any given $\Gamma$. Each side $s$ of $R \cap D$ is identified with another side $s^{\prime}$, by an element $g(s) \in \Gamma$. The set $\{g(s): s \in \partial R\}$ forms a symmetric set of generators for $\Gamma([6], \S 23)$.

Let $v_{1}$ be a vertex of $R$ and $s_{1}$ an adjacent side; then $v_{2}=g\left(s_{1}\right)\left(v_{1}\right)$ is another vertex and $s_{2}=g\left(s_{1}\right) s_{1}$ an adjacent side. Let $s_{2}^{\prime}$ be the other side of $R$ adjacent to $s_{2}$. Let $v_{3}=g\left(s_{2}^{\prime}\right) v_{2}, s_{3}=g\left(s_{2}^{\prime}\right) s_{2}^{\prime}$, and so on. Eventually $\left(v_{n+1}, s_{n+1}^{\prime}\right)=\left(v_{1}, s_{1}\right)$. Then $g\left(s_{n}^{\prime}\right) \cdots g\left(s_{1}\right)$ fixes $v_{1}$. If $v_{1} \in D, g\left(s_{n}^{\prime}\right) \cdots g\left(s_{1}\right)$ is elliptic and has order $\nu \in \mathbb{N}$; otherwise, if $v_{1} \in S^{1}$, it is parabolic $([6], \S 27)$. The relations $\left[g\left(s_{n}^{\prime}\right) \cdots g\left(s_{1}\right)\right]^{\nu}=1$ for all elliptic vertices $v$, form a complete set of relations for $\Gamma$ [7].

The graph $G(\Gamma)$ of $\Gamma$ may be represented as a net in $D$. The vertices are the points $g 0, g \in \Gamma$ (we may clearly assume 0 is not an elliptic fixed point so that $g \mapsto g 0$ is bijective), and the edges are the directed lines joining vertices $g 0, g^{\prime} 0$ for which $g^{-1} g^{\prime} \in \Gamma_{0}$. Such an edge we label $g^{-1} g^{\prime}$.

Relations in $\Gamma$ correspond to closed paths in $G(\Gamma)$. Regions bounded by edges of $G(\Gamma)$ with no edges intersecting the interior we call polygons. If $L$ is a region bounded by edges we write $|L|$ for the number of polygons in $L$, and $\partial L$ for the boundary of $L$. If $S \subseteq \partial L$ is a union of edges we write $|S|$ for the number of edges in $S$.

We always label arcs on $S^{1}$ in the anticlockwise direction, so that $[P Q]$ or $(P Q)$ means the closed or open interval of points between $P$ and $Q$ moving in an anticlockwise direction. We write $|P Q|$ for the length of the arc $[P Q]$.

\section{The graph of $\Gamma$}

Suppose $\Gamma$ is a finitely generated Fuchsian group with a symmetrical set of generators $\Gamma_{0}$ obtained from a fundamental region $R$ as described above. The relations in $\Gamma$ are of the form

$$
C_{i}=e_{i_{1}} \cdots e_{i_{r_{i}}}, \quad i=1, \ldots, k, \quad e_{i} \in \Gamma_{0} .
$$

Any occurrence of generators which occur consecutively in the same order in some $C_{i}$ we shall call a cycle. An occurrence of more than $\left[r_{i} / 2\right]$ elements of $C_{i}$ in order we call a long cycle. Occurrences of $r_{i}-1$ or $r_{i}-2$ elements in order we shall denote $F$ or $F^{-}$cycles respectively.

Any $g \in \Gamma$ has many representations $g=e_{1} \ldots e_{n}, e_{i} \in \Gamma_{0}$. We call such a representation shortest if it contains a minimum number of generators. Shortest representations obviously contain no occurrences $e e^{-1}, e \in \Gamma_{0}$. Moreover, they contain no long cycles, for a cycle in $C_{i}$ of length greater than $\left[r_{i} / 2\right]$ can be replaced by a cycle of length less than $\left[r_{i} / 2\right]$, using the relation $C_{i}=1$.

By a chain in $G(\Gamma)$ we mean a sequence of polygons $P_{j}, 1 \leq j \leq n$, such that $\left|\partial P_{j} \cap \partial P_{j+1}\right|=1,1 \leq j<n$, and such that $P_{j} \cap P_{k}=\varnothing, k-j \geq 2$, unless possibly if $P_{j+1}, P_{j+2}, \ldots, P_{k-1}$ all have three or four sides. Let $L$ be a chain and let $V, W$ be vertices of $P_{1}, P_{n}$ not lying in $P_{2}$ or $P_{n-1}$. There are two paths in $\partial L$ joining $V$ to 


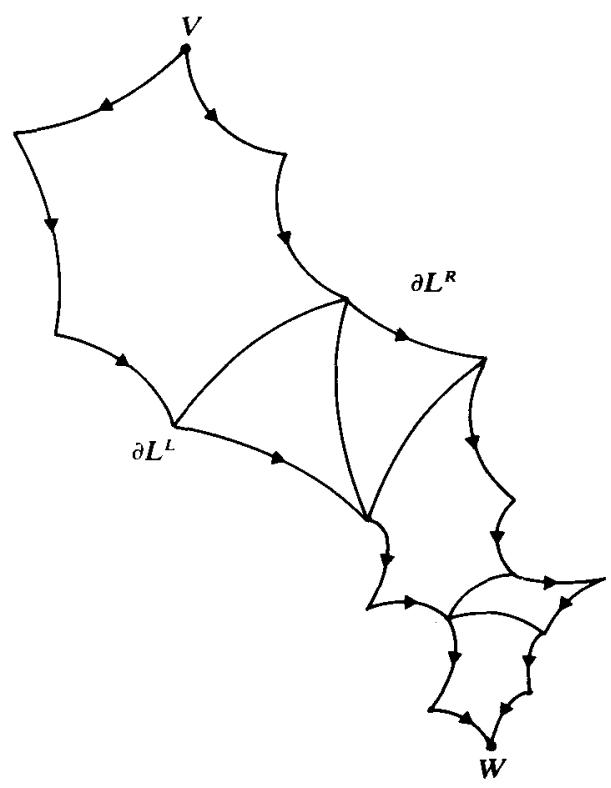

FIGURE 2

$W$. We call the clockwise path from $V$ to $W$ the right boundary, denoted $\partial L^{R}$, and the anticlockwise path the left boundary, denoted $\partial L^{L}$, see figure 2 .

In this section we show that, except for a small number of exceptional groups $\Gamma$, any two distinct shortest representations of $g \in \Gamma$ are opposite boundaries of a chain in $G(\Gamma)$. The argument follows the ideas of Dehn [4]. We begin with the assumption that at least five edges meet at a vertex of $G(\Gamma)$ and that all polygons in $G(\Gamma)$ have at least five sides. We shall then modify the argument to cover certain other groups with smaller numbers of generators or shorter cycles, and to include all non-co-compact groups.

LEMMA 2.1. Let $L \subseteq G(\Gamma)$ be a bounded simply connected union of polygons, so that $\partial L$ has no self-intersections. Then there is a polygon $P \subseteq L$ so that $\partial P \cap \partial L$ has exactly one connected component.

Proof. Suppose the result is not true. $\partial L$ is topologically a circle and is the union of the connected components of $\partial P \cap \partial L$ for polygons $P \subseteq L$. Join components $C$, $C^{\prime}$ corresponding to the same polygon $P$ by an arc $A\left(C, C^{\prime}\right)$ lying in $P-\left\{P^{\prime} \subseteq L, P \neq P^{\prime}\right\}$. To each component $C$ is associated at least one other component belonging to the same polygon $P$. Therefore at least one pair of $\operatorname{arcs} A, A^{\prime}$ lying in distinct polygons $P, P^{\prime}$ must intersect, which is impossible.

MAIN Lemma 2.2. Suppose that in $G(\Gamma)$ at least five edges meet at a vertex. Let $L$ be a simply connected region in $G(\Gamma)$ so that $\partial L$ has no self-intersections. Then either:

(i) $|L|=1$; or

(ii) $L$ is a chain and the two end polygons have $F$ cycles in $\partial L$; or

(iii) $\partial L$ contains at least three disjoint cycles of length $F^{-}$. 


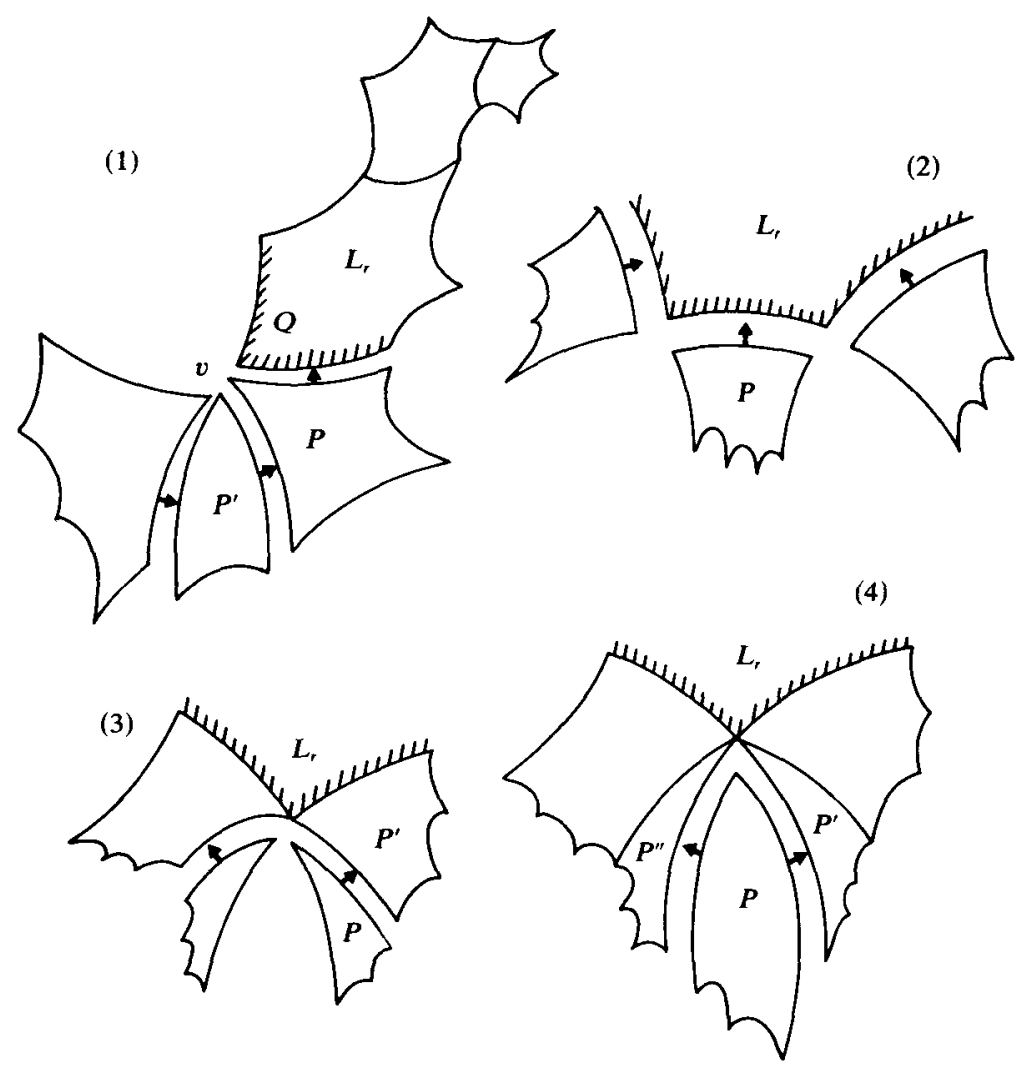

Figure 3

Remark. For later convenience, we mark the points in the proof which require particular hypotheses on $\Gamma$ with a superscript ${ }^{(n)}$.

Proof. The steps are illustrated in figure 3.

Let $L_{0} \subset L$ be a polygon chosen as in lemma 2.1 such that $\partial L_{0} \cap \partial L$ has one connected component. Let $L_{1}$ be the union of all polygons in $L$ which have a vertex in common with $L_{0}$, but excluding $L_{0}$. Let $R_{1}=L_{0} \cup L_{1}$. Inductively define $L_{n}$ to be the union of all polygons in $L$ with a vertex in common with $L_{n-1}$, but not contained in $R_{n-2}$, and let $R_{n}=L_{n} \cup R_{n-1}$. Then $L=\bigcup_{i=0}^{N} L_{i}$, say.

It is clear that if $\left|L_{i}\right|=1, i=1, \ldots, N$, then $L$ is a chain and (ii) holds. Otherwise, suppose $\left|L_{i}\right|=1, i=0, \ldots, r$, but $\left|L_{r+1}\right|>1$. Choose a polygon $P \subset L_{r+1}$ so that $S=R_{r} \cup P$ is a chain. Now choose another polygon $P^{\prime} \subset L_{r+1}$ so that $P^{\prime} \cap S \neq \varnothing$. Since more than four edges of $G(\Gamma)$ meet at a vertex, ${ }^{(1)} P^{\prime}$ can be attached to $S$ along only one edge.

In adding $P^{\prime}$ we reduce an $F$ cycle in $\partial S$ to an $F^{-}$cycle and add an $F$ cycle in $\partial P^{\prime}$.

We now build up $L$ by successively adding all polygons in $L_{r+1}$, then all in $L_{r+2}$, and so on. To form $L_{r+1}$, we first add all polygons with an edge in common with 
$L_{r}$, then those with only a vertex in $L_{r}$, making sure that at each stage the polygon $P$ we add has at least one edge attached to the previous figure $L^{\prime}$.

It is clear that in adding a polygon $P$ to an edge of $L_{r}$ we destroy at most one $F^{-}$cycle in $\partial L^{\prime}$ and add an $F$ cycle in $\partial\left(P \cup L^{\prime}\right){ }^{(2)}$ (This uses the fact that more than three edges of $G(\Gamma)$ meet at a vertex.)

If we add a polygon $P$ attached to $L_{r}$ at one vertex and to a polygon $P^{\prime} \subset L_{r+1}$ which has an edge in common with $L_{r}$, then we destroy at most one $F^{-}$cycle in $\partial P^{\prime} \cap \partial L^{\prime}$ but add an $F$ cycle in $\partial P^{(3)}$ (This uses that more than four edges meet at a vertex.)

Since at least five edges of $G(\Gamma)$ meet at a vertex, ${ }^{(4)}$ the only other possibility is that we add a polygon $P$ attached to $L_{r}$ at one vertex $V$ in such a way that the edges of $P$ at this vertex are one or both attached to polygons $P^{\prime}, P^{\prime \prime} \subset L_{r+1}$, such that at most one of $P^{\prime}, P^{\prime \prime}$ has an edge in $L_{r}$. Therefore we add at least an $F^{-}$cycle in $\partial P$ and remove at most one from $\partial L^{\prime}$, in the polygon $P^{\prime}$ which was joined to $L_{r}$ along an edge.

COROLlARY 2.3. If at least five edges meet at a vertex of $G(\Gamma)$ and every polygon in $G(\Gamma)$ has at least five sides, then the boundary of any bounded simply connected region $L$ with $\partial L$ non-self-intersecting contains at least three long cycles, unless $L$ is a chain.

We now allow an arbitrary number (necessarily $\geq 3$ ) of edges at a vertex in $G(\Gamma)$.

LEMMA 2.4. Suppose that in $G(\Gamma)$ at least three of the polygons meeting at a vertex have at least seven sides. Let $L$ be a bounded simply connected region in $G(\Gamma)$ so that $\partial L$ has no self-intersections. Then either:

(i) $|L|=1$; or

(ii) $L$ is a chain; or

(iii) $\partial L$ contains at least three disjoint long cycles.

Proof. We copy the proof of 2.2. Starting with $L_{0}$, continue forming regions $R_{0}, R_{1}, \ldots$ until $R_{r}$ is a chain but $R_{r+1}$ is not. Add $P \subset L_{r+1}$ so that $S=R_{r} \cup P$ is a chain as before.

Suppose the adjacent polygon in $R_{r}$ to $P$ is $Q$. Now take $P^{\prime} \subset L_{r+1}$, attached to $R, \cup P$ along at least one edge.

If $P^{\prime}$ is attached only along an edge of $Q$ we have obviously added an $F$ cycle and destroyed nothing. If $\boldsymbol{P}^{\prime}$ is attached only along an edge of $\boldsymbol{P}$ with a vertex $V \in Q$, we have either continued the chain, if $|\partial P|=3$ or 4 , or we have not destroyed anything. If only three edges meet at a vertex, then $\boldsymbol{P}^{\prime}$ may be attached along an edge of $P$ and of $Q$. But in this case, as all polygons have at least seven sides, we have added an $F^{-}$cycle and reduced an $F$ cycle to an $F^{-}$cycle.

Now if $|\partial P|=3$ or $4, P^{\prime}$ continued the chain. Repeat the above argument with another polygon $P^{\prime \prime} \subset L_{r+1}$, keeping the same vertex $V$. Again either $\left|\partial P^{\prime}\right|=3$ or 4 , and we continue the chain; or we add an $F$ cycle and destroy nothing, or $P^{\prime \prime}$ is attached along an edge of $P^{\prime}$ and of $Q$. In the last case, four edges meet at $V$ and since $|\partial P|=3,\left|\partial P^{\prime}\right|$ and $\left|\partial P^{\prime \prime}\right|$ are greater than seven so adjoining $P^{\prime \prime}$ adds an $F^{-}$ 
cycle and destroys nothing. In general, it is clear that we can continue adjoining 3 or 4 sided polygons at $V$ until we reach the last two which both have more than 7 sides, so we have finished. This deals with (1) in lemma 2.2.

We now proceed building up $L$ as in lemma 2.2 , but assume only that at each stage the figure we have is either a chain or its boundary contains at least three long cycles.

At (2) there is only a problem if only three edges meet at a vertex. Since then all cycles have length at least seven, adjoining a polygon destroys at most one long cycle and adds another.

The situation of (3) does not arise with only three edges at a vertex. If there are four edges at a vertex, the added polygon $P$ may be attached along two edges to polygons $P^{\prime}, P^{\prime \prime}$ in $L_{r+1}$, say. But then at most one of $|\partial P|,\left|\partial P^{\prime}\right|<7$, so adding $P$ destroys at most one long cycle; and in this case $|\partial P| \geq 7$ so a new long cycle is added.

Finally at (4), the situation only arises when at least five edges meet at $V$, and all polygons meeting at $V$ are attached. Let the polygons at $V$ in $L_{r+1}$ with edges in $L_{r}$ be $P^{\prime}, P^{\prime \prime}$. Attaching the remaining polygons at $V$ we destroy zero, one, or two long cycles in $\partial P^{\prime}, \partial P^{\prime \prime}$ according as neither, one, or both of $P^{\prime}, P^{\prime \prime}$ has less than seven sides. Of the $m$ polygons we add, in these cases at least zero, one or two have seven or more sides respectively, so that we always add at least enough long cycles to compensate for the ones removed.

LEMMA 2.5. If $\Gamma$ is a non-co-compact group then any bounded simply connected region with non-self-intersecting boundary is either a chain or contains at least three F cycles.

Proof. The condition $\Gamma$ non-co-compact means that no vertex of $G(\Gamma)$ is in the interior of $L$, because $L$ is bounded and at each vertex of $G(\Gamma)$ is at least one polygon with an infinite number of sides.

We build up chains just as in lemma 2.2. All problems arise when the polygons we attach are attached to more than one edge of the previous figure. But if we follow the procedure in 2.2 we note that this can now never happen.

Remark 2.6. The reader may legitimately ask about the groups not included in the above lemmas. But it is easy to see that for at least some of these groups, there are non-chains of arbitrary large size whose boundaries contain no long cycles. See, for example, figures 4 and 5 .

From now on we shall refer to graphs satisfying the hypotheses of any of corollary 2.3 , or lemma 2.4 or 2.5 as non-exceptional.

What we have proved is:

THEOREM 2.7. Let $\Gamma$ be any finitely generated Fuchsian group with non-exceptional graph $G(\Gamma)$. Let $L \subset G(\Gamma)$ be any bounded simply connected region so that $\partial L$ has no self-intersections, and so that $L$ contains at most two long cycles. Then $L$ is a polygon or a chain. 


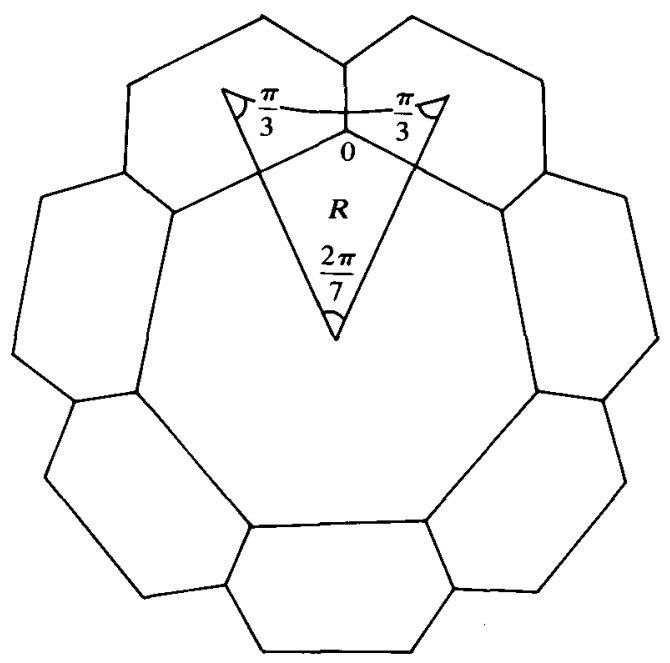

FIGURE 4

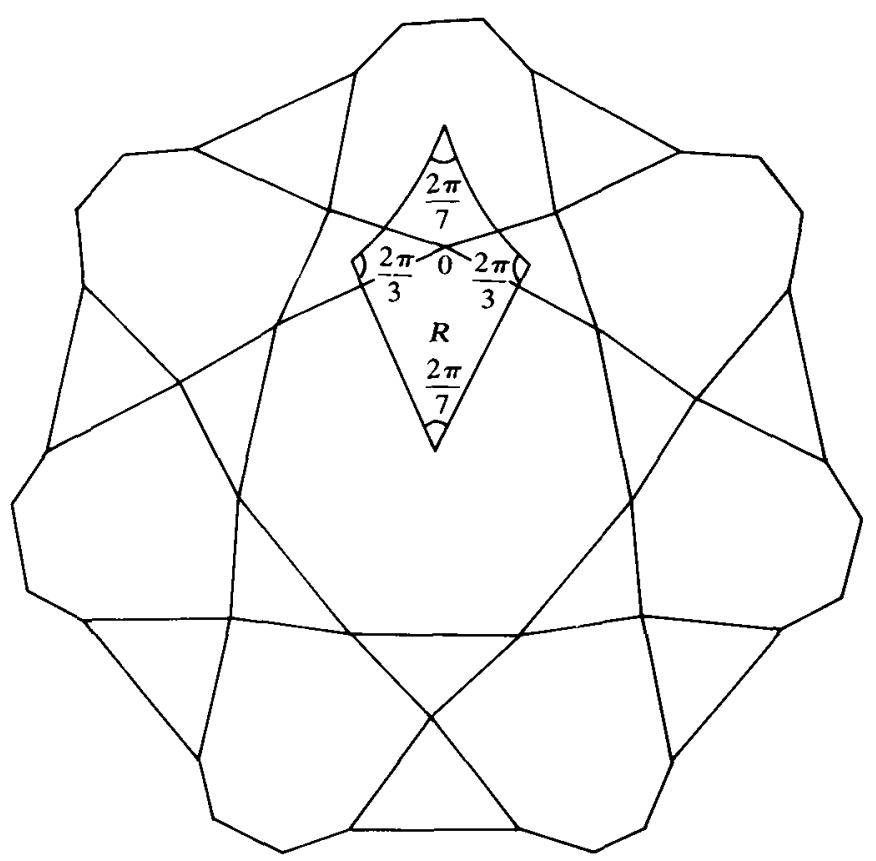

FIGURE 5

It is easy to deduce from this result that any two distinct shortest representations of $g \in \Gamma$ must be opposite boundaries of a chain. This fact is exploited in detail in the next section.

3. The word problem

From now on we restrict our attention entirely to non-exceptional graphs. We specify a set of rules for admissible sequences of generators in $\Gamma_{0}$ and show that 
any element in $\Gamma$ has a unique shortest admissible representation as a product of generators in $\Gamma_{0}$.

We keep to the notation and terminology introduced above. A cycle $E$ always consists of a connected part of the boundary of a polygon in $G(\Gamma)$. If the generators occur in clockwise order round $P$ we call $E$ a right cycle; otherwise a left cycle. Suppose $P, Q$ are polygons with a common edge, and that $A \subseteq \partial P, B \subseteq \partial Q$ are both right or both left cycles in $\partial P, \partial Q$ with one common vertex (the end of $A$ and the beginning of $B$ ). Then $A$ and $B$ are called consecutive right or left cycles. It is clear that the right and left boundaries of a chain are sequences of consecutive right and left cycles (we include the case when the chain contains three or four sided polygons which meet one side of the chain only in a point).

The point of the next definition is to give a means of identifying the boundaries of certain special chains which correspond to shortest paths in $G(\Gamma)$.

Definition 3.1. A sequence of consecutive right cycles $A_{1} A_{2} \cdots A_{n}$, where $A_{i}$ lies in the boundary of a polygon with $r_{i}$ sides, is called extreme if:

(i) $\left|A_{1}\right|=\frac{1}{2} r_{1}$ if $r_{1}$ is even, $\frac{1}{2}\left(r_{1}-1\right)$ otherwise;

(ii) $\left|A_{i}\right|=\frac{1}{2}\left(r_{i}-2\right)$ if $r_{i}$ is even, or $\frac{1}{2}\left(r_{i} \pm 1\right)-1$ if $r_{i}$ is odd, for $i>1$; and

(iii) $0 \leq \sum_{i=1}^{r} \sigma\left(A_{i}\right) \leq 1,1 \leq i \leq n$, where $\sigma\left(A_{1}\right)=1$ if $r_{1}$ is even, 0 otherwise, $\sigma\left(A_{i}\right)=0$ if $r_{i}$ is even, \pm 1 if $r_{i}$ is odd, and $\left|A_{i}\right|=\frac{1}{2}\left(r_{i} \pm 1\right)-1, i>1$.

A sequence $A_{1} \cdots A_{n} x$, where $A_{1} \cdots A_{n}$ is an extreme right sequence and $x \in \Gamma_{0}$ is the element next following $A_{n}$ in right-hand cyclic order is called an excessive right sequence.

A sequence of consective left cycles $B_{1} \cdots B_{n}$, where $B_{i}$ is part of a polygon of $r_{i}$ sides, is called extreme if:

(i) $\left|B_{1}\right|=\frac{1}{2} r_{1}-1$ if $r_{1}$ is even, $\frac{1}{2}\left(r_{1}-1\right)$ otherwise;

(ii) $\left|B_{i}\right|=\frac{1}{2}\left(r_{i}-2\right)$ if $r_{i}$ is even, or $\frac{1}{2}\left(r_{i} \pm 1\right)-1$ if $r_{i}$ is odd, for $i>1$; and

(iii) $-1 \leq \sum_{i=1}^{r} \tau\left(B_{i}\right) \leq 0,1 \leq i \leq n$, where $\tau\left(B_{1}\right)=-1$ if $r_{1}$ is even, 0 otherwise and $\tau\left(B_{i}\right)=0$ if $r_{i}$ is even and \pm 1 if $r_{i}$ is odd and $\left|B_{i}\right|=\frac{1}{2}\left(r_{i} \pm 1\right)-1$.

A sequence $B_{1} \cdots B_{n} x$, where $B_{1} \cdots B_{n}$ is an extreme left sequence and $x$ follows $B_{n}$ in left-hand cyclic order, is called an excessive left sequence.

We can now state the rules for admissible sequences as:

Definition 3.2. A sequence $e_{1} \cdots e_{n}$ of generators in $\Gamma_{0}$ is called admissible if:

(i) $e e^{-1}, e \in \Gamma_{0}$, never occurs;

(ii) there are no blocks consisting of excessive right- or left-hand sequences.

The idea of these rules is that, whenever there is a choice of shortest paths, the paths bound a chain and we choose the right-hand boundary.

One verifies immediately that an admissible sequence contains no long cycles and no left cycle of length $\frac{1}{2}\left|C_{i}\right|$. Thus we have in particular chosen the right-hand path round a polygon. If $e_{1} \cdots e_{n}$ is any path in $G(\Gamma)$ we may without increasing the length replace it by a path in which there are no cancellations $e e^{-1}$, no long cycles, and no left cycles of length $\frac{1}{2}\left|C_{i}\right|$. Such a representation we call standard. 
LeMMA 3.3. Let $e_{1} \cdots e_{r}$ be an admissible sequence. Then $e_{1} \cdots e_{r}$ is a shortest representation of $g=e_{1} \cdots e_{r}$.

Proof. We can regard the sequence of directed edges $e_{1}, \ldots, e_{r}$ from 0 to $g 0$ as a path in $G(\Gamma)$. Suppose that $f_{1} \cdots f_{t}$ is also a path from 0 to $g 0$ and that $t \leq r$. We may assume $f_{1} \cdots f_{t}$ is in standard form. We may also assume $e_{1} \neq f_{1}$ (otherwise keep cancelling on the left until $\left.e_{i} \neq f_{i}\right)$. Suppose that $f_{1} \cdots f_{p}=e_{1} \cdots e_{s}$ but $f_{1} \cdots f_{q} \neq e_{1} \cdots e_{t}$ for $q+t<p+s$. Then the paths $e_{1} \cdots e_{s}, f_{1} \cdots f_{p}$ from 0 to $h 0$, $h=e_{1} \cdots e_{s}$, only meet at 0 and $h 0$. Apply theorem 2.7 to the region $L$ enclosed by these paths. Since $e_{1} \cdots e_{s}, f_{1} \cdots f_{p}$ are in standard form $\partial L$ can contain at most two long cycles and is therefore a chain $\bigcup_{i=1}^{N} P_{i}$. Write $\partial L^{R}, \partial L^{L}$ for the clockwise and anti-clockwise paths from 0 to $h 0$ respectively, and $\partial P_{i}^{R}=\partial P_{i} \cap \partial L^{R}, \partial P_{i}^{L}=$ $\partial P_{i} \cap \partial L^{L}$.

Suppose first $f_{1} \cdots f_{q}$ is $\partial L^{R}$ and $e_{1} \cdots e_{s}$ is $\partial L^{L}$. Then

$$
\left|\partial P_{1}^{R}\right| \leq \frac{1}{2}\left|\partial P_{1}\right| \quad \text { if }\left|\partial P_{1}\right| \text { is even }
$$

and

$$
\left|\partial P_{1}^{R}\right| \leq \frac{1}{2}\left(\left|\partial P_{1}\right|-1\right) \quad \text { if }\left|\partial P_{1}\right| \text { is odd, }
$$

because otherwise $f_{1} \cdots f_{p}$ is not a shortest path. Likewise, since $e_{1} \cdots e_{s}$ is admissible,

$$
\left|\partial P_{1}^{L}\right| \leq \frac{1}{2}\left|\partial P_{1}\right|-1 \quad \text { if }\left|\partial P_{1}\right| \text { is even }
$$

and

$$
\left|\partial P_{1}^{L}\right| \leq \frac{1}{2}\left(\left|\partial P_{1}\right|-1\right) \quad \text { otherwise. }
$$

Adding, and using $\left|\partial P_{1}^{R}\right|+\left|\partial P_{1}^{L}\right|=\left|\partial P_{1}\right|-1$, we see we have equality in all cases. Thus both $\partial P_{1}^{R}, \partial P_{1}^{L}$ are extreme, and

$$
\left|\partial P_{1}^{R}\right|-\left|\partial P_{1}^{L}\right|=\frac{1}{2}\left|\sigma\left(\partial P_{1}^{R}\right)-\tau\left(\partial P_{1}^{L}\right)\right|
$$

Let

$$
\partial L_{r}^{R}=\partial P_{1}^{R} \cup \cdots \cup \partial P_{r}^{R}, \quad \partial L_{r}^{L}=\partial P_{1}^{L} \cup \cdots \cup \partial P_{r}^{L},
$$

and let

$$
\sigma\left(\partial L_{r}^{R}\right)=\sum_{i=1}^{r} \sigma\left(\partial P_{i}^{R}\right), \quad \tau\left(\partial L_{r}^{L}\right)=\sum_{i=1}^{r} \tau\left(\partial P_{i}^{L}\right) .
$$

Now assume inductively that for $1 \leq k<r<n, \partial L_{k}^{R}$ and $\partial L_{k}^{L}$ are extreme; and that

$$
\left|\partial L_{k}^{R}\right|-\left|\partial L_{k}^{L}\right|=\frac{1}{2}\left|\sigma\left(\partial L_{k}^{R}\right)-\tau\left(\partial L_{k}^{L}\right)\right| .
$$

In particular, we have

$$
\left|\partial L_{r-1}^{R}\right|-\left|\partial L_{r-1}^{L}\right|=1 \text { or } 0 .
$$

Suppose first $\left|\partial P_{r}\right|$ is even. Since $f_{1} \cdots f_{p}$ is shortest, and

$$
\left|\partial L_{r-1}^{R}\right| \geq\left|\partial L_{r-1}^{L}\right|+1, \quad\left|\partial P_{r}^{R}\right| \leq \frac{1}{2}\left|\partial P_{r}\right|-1 .
$$

Since $e_{1} \cdots e_{s}$ is admissible,

$$
\left|\partial P_{r}^{L}\right| \leq \frac{1}{2}\left|\partial P_{r}\right|-1
$$


Adding, we see we have both equalities. It is now easy to check that the inductive step follows.

Suppose $\left|\partial P_{r}\right|$ is odd. A similar argument shows that if

$$
\begin{gathered}
\left|\partial L_{r-1}^{R}\right|-\left|\partial L_{r-1}^{L}\right|=1 \\
\left|\partial P_{r}^{R}\right|=\frac{1}{2}\left(\left|\partial P_{r}\right|-3\right) \text { and }\left|\partial P_{r}^{L}\right|=\frac{1}{2}\left(\left|P_{r}\right|-1\right) ;
\end{gathered}
$$

and that otherwise

$$
\left|\partial P_{r}^{R}\right|=\frac{1}{2}\left(\left|\partial P_{r}\right|-1\right) \text { and }\left|\partial P_{r}^{L}\right|=\frac{1}{2}\left(\left|\partial P_{r}\right|-3\right) .
$$

One checks again that the $\sigma$ s and $\tau$ s work.

Finally, adding $P_{n}$ we obtain a contradiction: if $\left|\partial L_{n-1}^{R}\right|-\left|\partial L_{n-1}^{L}\right|=0$ we have $\sigma\left(\partial L_{n-1}^{R}\right)=\tau\left(\partial L_{n-1}^{L}\right)=0$.

Suppose $\left|\partial P_{n}\right|$ is even. Since $\partial L^{L}$ is admissible, $\left|\partial P_{n}^{L}\right| \leq \frac{1}{2}\left(\left|\partial P_{n}\right|-2\right)$. Since $\partial L^{R}$ is shortest, $\left|\partial P_{n}^{R}\right| \leq \frac{1}{2}\left|\partial P_{n}\right|$. Adding, we must have equality. But then $\left|\partial L^{R}\right|>\left|\partial L^{L}\right|$ which is not the case.

If $\left|\partial P_{n}\right|$ is odd, $\left|\partial P_{n}^{L}\right| \leq \frac{1}{2}\left(\left|\partial P_{n}\right|-3\right)$ and $\left|\partial P_{n}^{R}\right| \leq \frac{1}{2}\left(\left|\partial P_{n}\right|-1\right)$. Adding gives a contradiction.

A similar argument works if $\left|\partial L_{n-1}^{R}\right|-\left|\partial L_{n-1}^{L}\right|=1$.

We conclude $e_{1} \cdots e_{s}$ was a right-hand boundary. Going through an exactly similar argument again shows that $e_{1} \cdots e_{s}$ is shortest.

LEMMA 3.4. Let $f_{1} \cdots f_{s}$ be any sequence which is a shortest path from 0 to g0, $g=f_{1} \cdots f_{s}$. Then there is an admissible sequence $e_{1} \cdots e_{s}$ with $e_{1} \cdots e_{s}=g$.

Proof. We may assume $f_{1} \cdots f_{s}$ is in standard form. If it is not admissible it must contain an excessive sequence of consecutive cycles. This sequence forms one boundary of a chain of polygons $P_{1}, \ldots, P_{n}$ joining $V \in P_{1}$ to $W \in P_{n}$. Keeping the notation of lemma 3.3, one verifies that $\partial L_{r}^{R}, \partial L_{r}^{L}$ are both extreme paths and that

$$
\left|\partial L_{r}^{R}\right|-\left|\partial L_{r}^{L}\right|=\frac{1}{2}\left|\sigma\left(\partial L_{r}^{R}\right)-\tau\left(\partial L_{r}^{L}\right)\right|, \quad 1 \leq r<n .
$$

Suppose first that the excessive sequence in $f_{1} \cdots f_{s}$ is $\partial L_{n}^{R}$. Then $\sigma\left(\partial L_{n-1}^{R}\right)=1$, $\left|\partial P_{n}\right|$ is odd and $\left|\partial P_{n}^{R}\right|=\frac{1}{2}\left(\left|\partial P_{n}\right|-1\right)$. But then $\left|\partial L_{n}^{R}\right|-\left|\partial L_{n}^{L}\right|=1$ so $f_{1} \cdots f_{s}$ was not shortest.

So the excessive sequence must be the boundary $\partial L_{n}^{L}$. Then $\sigma\left(\partial L_{n-1}^{L}\right)=0,\left|\partial P_{n}\right|$ is odd and $\left|\partial P_{n}^{L}\right|=\frac{1}{2}\left(\left|\partial P_{n}\right|-1\right)$. But then $\left|\partial L_{n}^{R}\right|=\left|\partial L_{n}^{L}\right|$ and $\partial L_{n}^{R}$ is extreme, so we can replace $\partial L_{n}^{L}$ with the equal admissible path $\partial L_{n}^{R}$.

THEOREM 3.5. If a finitely generated Fuchsian group $\Gamma$ has non-exceptional graph $G(\Gamma)$, then every $g \in \Gamma$ has a unique shortest admissible representation as a product of generators in $\Gamma_{0}$.

Proof. By lemmas 3.3 and 3.4 it is enough to show that, if $e_{1} \cdots e_{s}=f_{1} \cdots f_{s}$ are both shortest admissible sequences, then they are equal. By the argument of lemma 3.3 we may restrict to sequences $e_{1} \cdots e_{s}=f_{1} \cdots f_{s}$ where $e_{1} \cdots e_{p} \neq f_{1} \cdots f_{r}, p+r<$ 2s. As in that lemma these sequences bound a chain $L=\bigcup_{i=0}^{N} P_{i}$. Following the argument in 3.3, we see that, since $f_{1} \cdots f_{s}$ and $e_{1} \cdots e_{s}$ are both shortest and both admissible, they must both be $\partial L^{R}$. But then they are equal. 
4. Infinite words and the limit set

We keep to our assumption that $\Gamma$ has a set of generators $\Gamma_{0}$ with non-exceptional graph $G(\Gamma)$. We call a word $x_{1} \cdots x_{n}, x_{i} \in \Gamma_{0}$, admissible, if it satisfies definition 3.2.

Let $\Sigma_{F}=\left\{x_{1} \cdots x_{n}: x_{i} \in \Gamma_{0}, x_{1} \cdots x_{n}\right.$ is admissible $\}$. By theorem 3.5, the natural $\operatorname{map} \pi_{F}: \Sigma_{F} \rightarrow D, \pi_{F}\left(x_{1} \cdots x_{n}\right)=x_{1} \cdots x_{n} 0$, is a bijection onto $\Gamma 0$.

Let

$$
\Sigma=\left\{\left(x_{i}\right)_{i=1}^{\infty}: x_{1} \cdots x_{n} \in \Sigma_{F} \text { for all } n\right\}
$$

We aim to extend $\pi_{F}$ to a map $\pi: \Sigma \rightarrow \Lambda$, the limit set of $\Gamma$.

For $x=x_{1} \cdots x_{n} \in \Sigma_{F}$ write $|x|=n$. For $u, v \in D$ let $H(u, v)$ be the hyperbolic distance of $u, v$ and $E(u, v)$ the Euclidean distance.

Proposition 4.1. (i) If $\Gamma$ has no parabolic elements, there is a constant $\alpha>0$ so that

$$
H(0, x 0)>\alpha|x| \text { for } x \in \Sigma_{F} \text {. }
$$

(ii) If $\Gamma$ has parabolic elements, there are $k, n_{0} \in \mathbb{N}$ so that

$$
H(0, x 0)>2 \log |x|-k \text {, whenever } x \in \Sigma_{F},|x|>n_{0} .
$$

Proof. This is [5], $\$ 4$.

Put a metric $\rho$ on $\Sigma_{F}$ as follows:

if $\Gamma$ has no cusps, $\rho(x, y)=\exp (-n)$ if $x_{i}=y_{i}, i \leq n, x_{n+1} \neq y_{n+1}$;

if $\Gamma$ has cusps, $\rho(x, y)=n^{-2}$ if $x_{i}=y_{i}, i \leq n, x_{n+1} \neq y_{n+1}$.

Proposition 4.2. The map $\pi_{F}: \Sigma_{F} \rightarrow D$ is continuous. If $\Gamma$ has no cusps, $E(x 0, y 0) \leq$ $\gamma \rho(x, y)^{\alpha}$ for some $\gamma, \alpha>0$.

Proof. The formula $d s=2|d z| /\left(1-|z|^{2}\right)$ for the hyperbolic metric gives

$$
E(0, P)=\tanh \frac{H(0, P)}{2}, \quad P \in D .
$$

Let $D_{r}=\{z \in \mathbb{C}:|z| \leq r\}, r<1$, and suppose the geodesic arc connecting $P, Q \in D$ lies entirely outside $D_{r}$. Then

$$
E(P, Q) \leq \frac{1}{2}\left(1-r^{2}\right) H(P, Q) .
$$

Since hyperbolic circles are convex (they are off-centre Euclidean circles), if $H(0, P)>L$ then $B_{K}^{H}(P) \subseteq B_{\varepsilon}^{E}(P)$, where $B_{K}^{H}, B_{\varepsilon}^{E}$ are hyperbolic and Euclidean balls of radius $K, \varepsilon$, and

$$
\varepsilon=\frac{1}{2} K \operatorname{sech}^{2} \frac{1}{2}(L-K) \leq c \exp (-L)
$$

where $c$ is independent of $L$.

Let $K=\max \left\{H(0, e 0): e \in \Gamma_{0}\right\}$. Suppose $\Gamma$ has no cusps and $\rho(x, y)=\exp (-n)$, $x=x_{1} \cdots x_{N}, y=y_{1} \cdots y_{M} \in \Sigma_{F}$. Then

$$
\begin{aligned}
E(x 0, y 0) \leq & \sum_{r=0}^{N-1} E\left(x_{1} \cdots x_{n+r} 0, x_{1} \cdots x_{n+r+1} 0\right) \\
& +\sum_{r=0}^{M-1} E\left(y_{1} \cdots y_{n+r} 0, y_{1} \cdots y_{n+r+1} 0\right) .
\end{aligned}
$$

By proposition 4.1,

$$
H\left(0, x_{1} \cdots x_{n+r} 0\right)>\alpha(n+r)
$$


and so

$$
B_{K}^{H}\left(x_{1} \cdots x_{n+r} 0\right) \subseteq B_{\beta}^{E}\left(x_{1} \cdots x_{n+r} 0\right), \quad \text { where } \beta=c \exp (-\alpha(n+r))
$$

Since $H\left(x_{1} \cdots x_{n+r} 0, x_{1} \cdots x_{n+r+1} 0\right) \leq K$,

$$
E\left(x_{1} \cdots x_{n+r} 0, x_{1} \cdots x_{n+r+1} 0\right) \leq c \exp (-\alpha(n+r)) .
$$

A similar relation holds for $y$. Thus $E(x 0, y 0) \leq \gamma \exp (-\alpha n)=\gamma \rho(x, y)^{\alpha}$ for suitable $\gamma$. If $\Gamma$ has cusps a similar argument gives

$$
E(x 0, y 0) \leq 2 c \sum_{r=0}^{\infty} \frac{1}{(n+r)^{2}}
$$

for sufficiently large $n$.

The metric $\rho_{F}$ extends to a metric $\rho$ on $\Sigma \cup \Sigma_{F}$ in a natural way and makes $\Sigma$ a compact metric space.

$\pi_{F}$ extends to $\pi: \Sigma \rightarrow \bar{D}$ and it is clear that $\pi(\Sigma) \subseteq \Lambda$.

We now investigate the map $\pi$ more closely. First observe:

Lemma 4.3. Suppose $g \in \Gamma_{0}, e=e_{1} e_{2} \cdots \in \Sigma$ and $g e_{1} e_{2} \cdots \in \Sigma$. Then $\pi\left(g e_{1} e_{2} \cdots\right)=$ $g \pi\left(e_{1} e_{2} \cdots\right)$. More generally, if $e_{1} e_{2} \cdots \in \Sigma, f_{1} f_{2} \cdots f_{m} e_{n+1} \cdots \in \Sigma$, and $h \in \Gamma$, $h e_{1} \cdots e_{n}=f_{1} \cdots f_{m}$, then $h \pi\left(e_{1} e_{2} \cdots\right)=\pi\left(f_{1} f_{2} \cdots\right)$.

Proof.

$$
\begin{aligned}
\pi\left(g e_{1} e_{2} \cdots\right) & =\lim _{n \rightarrow \infty} \pi\left(g e_{1} e_{2} \cdots e_{n} 0\right) \\
& =g \lim _{n \rightarrow \infty} \pi\left(e_{1} e_{2} \cdots e_{n} 0\right) \\
& =g \pi(e) .
\end{aligned}
$$

The second statement is proved similarly.

Any element $e=\left(e_{i}\right)_{i=1}^{\infty} \in \Sigma$ can be thought of as defining an infinite path $0, e_{1} 0, e_{1} e_{2} 0, \ldots$ in $G(\Gamma)$ which converges to $\pi(e) \in \Lambda$. Two paths, $e, f$ never meet in $D$ as this would contradict the uniqueness of representation of $g \in \Gamma$ by elements of $\Sigma_{F}$.

By an extreme right or an extreme left path in $\Sigma$ we mean a sequence $\left(e_{i}\right)_{i=1}^{\infty}$ such that every finite sequence $e_{1} \cdots e_{n}$, is an extreme right (or left) sequence whenever $e_{n}, e_{n+1}$ are elements of consecutive cycles; or is the right or left boundary of a polygon in $G(\Gamma)$ with an infinite number of sides. Geometrically this means that starting at 0 along the edge $e_{1}$, we turn as far as possible to the right (or left) at each stage, subject only to the constraint that the path be admissible. More generally, if $a_{1} \cdots a_{p}$ is an arbitrary sequence in $\Sigma_{F}$, we call the sequence $a_{1} \cdots a_{p} e_{1} \cdots \in \Sigma$ the extreme right path following $a_{1} \cdots a_{p}$ if at each stage after $a_{p}$ we turn as far as possible to the right (left) subject to the constraint that $a_{1} \cdots a_{p} e_{1} \cdots e_{n}$ be admissible. We denote the endpoints of the right and left extreme paths following $a_{1} \cdots a_{p}, \rho\left(a_{1} \cdots a_{p}\right)$ and $\lambda\left(a_{1} \cdots a_{p}\right)$ respectively.

For $e_{1} \cdots e_{n} \in \Sigma_{F}$, let $Z\left(e_{1} \cdots e_{n}\right)=\left\{x \in \Sigma: x_{1}=e_{1}, \ldots, x_{n}=e_{n}\right\}$, and let $I\left(e_{1} \cdots e_{n}\right)=\left[\rho\left(e_{1} \cdots e_{n}\right), \lambda\left(e_{1} \cdots e_{n}\right)\right]$. 
Proposition 4.4. $\pi\left(Z\left(e_{1} \cdots e_{n}\right)\right) \subseteq I\left(e_{1} \cdots e_{n}\right)$ whenever $e_{1} \cdots e_{n} \in \Sigma_{F \cdot}$

Proof. Let $a, b \in \Sigma$ be the extreme right- and left-hand paths following $0, e_{1} 0, \ldots, e_{1} \cdots e_{n} 0$ respectively. Suppose $e \in Z\left(e_{1} \cdots e_{n}\right)$. If $e$ coincides with $a$ or $b$ the result is obvious. Otherwise there exists $m>n$ so that $a_{i}=e_{i}, i \leq m$, and $a_{m+1} \neq e_{m+1}$. Since $a$ is an extreme right path, $e_{m+1}$ must point to the left of $a_{m+1}$. Having diverged from $a$, e cannot meet $a$ or $b$ again in $D$. This forces the result.

Proposition 4.5. $\left|I\left(e_{1} \cdots e_{n}\right)\right| \rightarrow 0$ as $n \rightarrow \infty$.

Proof. By proposition 4.2,

$$
E\left(\rho\left(e_{1} \cdots e_{n}\right), \lambda\left(e_{1} \cdots e_{n}\right)\right) \leq 2 c \sum_{r=0}^{\infty} 1 /(n+r)^{2}
$$

or

$$
E\left(\rho\left(e_{1} \cdots e_{n}\right), \lambda\left(e_{1} \cdots e_{n}\right)\right) \leq \gamma \exp (-\alpha n)
$$

according as $\Gamma$ has or has no cusps.

It is clear by an argument similar to that of proposition 4.4 that the intervals $I\left(e_{i}\right)$, $I\left(e_{i}\right), e_{i}, e_{i} \in \Gamma$ intersect in at most one point, and this only if $\lambda\left(e_{i}\right)=\rho\left(e_{j}\right)$ or vice versa. It is also clear that

$$
I\left(e_{1} \cdots e_{n}\right) \subseteq I\left(e_{1} \cdots e_{m}\right), \quad m<n,
$$

whenever $e_{1} \cdots e_{n} \in \Sigma_{F}$.

Proposition 4.6. $\pi$ is bijective except on pairs of points $e, f \in \Sigma$ such that $e_{i}=f_{i}$, $i \leq m, e_{m+1} \neq f_{m+1}, \rho\left(e_{m+1}\right)=\lambda\left(f_{m+1}\right)$ and such that $e, f$ coincide with the extreme right and left paths following $e_{1} \cdots e_{m+1}$ and $f_{1} \cdots f_{m+1}$.

Proof. It is clear that the images of such points coincide.

Suppose $\pi(e)=\pi(f), e, f \in \Sigma$, and suppose $e_{i}=f_{i}, i \leq m, e_{m+1} \neq f_{m+1}$. Then by proposition 4.4, $\pi\left(\sigma^{m} e\right) \in I\left(e_{m+1}\right)$ and $\pi\left(\sigma^{m} f\right) \in I\left(f_{m+1}\right)$.

By lemma 4.3, $\pi\left(\sigma^{m} e\right)=e_{m}^{-1} \cdots e_{1}^{-1} \pi(e)=\pi\left(\sigma^{m} f\right)$. Since $e_{m+1} \neq f_{m+1}$, one or other pair of endpoints of $I\left(e_{m+1}\right), I\left(f_{m+1}\right)$ must coincide at the point $\pi\left(\sigma^{m} e\right)$. Hence the result.

Let $Q=\bigcup_{a \in \Gamma_{0}} I(a)$ and define $f: Q \rightarrow Q$ by $f(x)=a^{-1} x$ for $x \in I(a)$.

If $I(a) \cap I(b) \neq \varnothing, a \neq b$, we allow $f$ to be two-valued at the common endpoint. It is clear that $\pi(\sigma e)=f(\pi(e)), e \in \Sigma$, provided that we take the appropriate value for $f$ at endpoints.

Proposition 4.7. $\Lambda \subseteq \bigcap_{n=0}^{\infty} f^{-n} Q$. (We always take the natural value for $f$ at endpoints.)

Proof. Since $\Lambda$ is $\Gamma$ invariant and $f$ is piecewise equal to elements of $\Gamma$ it is enough to show $\Lambda \subseteq Q$.

Suppose $x \in \Lambda, x \notin Q$. Let $\rho(a), \lambda(b)$ be the endpoints of the closest intervals $I(a), I(b)$ of $Q$ to $x$, so that $x \in(\lambda(a), \rho(b))$ and $(\lambda(a), \rho(b)) \cap Q=\varnothing$. Since $x \in \Lambda$ there is a point $g 0$ in the open region $T$ enclosed by the extreme left-hand path 
following $0, a 0$ (ending in $\lambda(a)$ ), the extreme right-hand path following $0, b 0$ (ending in $\rho(b)$ ), and the arc $[\lambda(a), \rho(b)]$. But then $g 0$ is connected to 0 by an admissible path which must lie entirely in $\bar{T}$. This is impossible, since such a path must start along $0, a 0$ or $0, b 0$ would have to lie to the left of the extreme left path from $a$ and the right of the extreme right path from $b$.

Proposition 4.8. (i) If $e_{0} \cdots e_{n} \in \Sigma_{F}$ then

$$
I\left(e_{0} \cdots e_{n-1}\right) \cap f^{-1} I\left(e_{1} \cdots e_{n}\right)=I\left(e_{0} \cdots e_{n}\right) \text {. }
$$

(ii) If $e_{0} \cdots e_{n-1} \in \Sigma_{F}$ but $e_{0} \cdots e_{n} \notin \Sigma_{F}$ then $f^{n} I\left(e_{0} \cdots e_{n-1}\right) \cap I\left(e_{n}\right)$ is either empty or consists of only one or other endpoint of $I\left(e_{n}\right)$.

Proof. (i) $f_{\mid I\left(e_{0} \cdots e_{n-1}\right)}=e_{0}^{-1}$. Suppose first $e_{0} \cdots e_{n}$ does not lie on the extreme left path following $0, e_{0} 0$. Since $\lambda\left(e_{0} \cdots e_{n}\right)$ is the end of the extreme left path following $0, e_{0} 0, \ldots, e_{0} \cdots e_{n} 0, f\left(\lambda\left(e_{0} \cdots e_{n}\right)\right)$ is the end of the extreme left path following $e_{0}^{-1} 0,0, e_{1} 0, \ldots, e_{1} \cdots e_{n} 0 . \lambda\left(e_{1} \cdots e_{n}\right)$ is the end of the extreme left path following $0, e_{1} 0, \ldots, e_{1} \cdots e_{n} 0$. Since $e_{0} \cdots e_{n}$ is not extreme, $f\left(\lambda\left(e_{0} \cdots e_{n}\right)\right)=\lambda\left(e_{1} \cdots e_{n}\right)$.

Applying similar reasoning to the right endpoints, it is clear that if $e_{0} \cdots e_{n}$ is neither the extreme right nor the extreme left path following $e_{0} \cdots e_{n-1}$, then

$$
f\left(I\left(e_{0} \cdots e_{n-1}\right)\right) \supseteq I\left(e_{1} \cdots e_{n}\right)
$$

and

$$
f^{-1} I\left(e_{1} \cdots e_{n}\right) \cap I\left(e_{0} \cdots e_{n-1}\right)=I\left(e_{0} \cdots e_{n}\right) .
$$

Now suppose $e_{0} \cdots e_{n}$ is the extreme left path following $0, e_{0} 0$. Then

$$
\lambda\left(e_{0} \cdots e_{n-1}\right)=\lambda\left(e_{0} \cdots e_{n}\right),
$$

and

$$
e_{0}^{-1} \lambda\left(e_{0} \cdots e_{n}\right) \in\left[\rho\left(e_{1} \cdots e_{n}\right), \lambda\left(e_{1} \cdots e_{n}\right)\right] .
$$

Since neither $e_{0} \cdots e_{n-1}$ nor $e_{0} \cdots e_{n}$ can be extreme right paths, by the reasoning above

$$
e_{0}^{-1} \rho\left(e_{0} \cdots e_{n}\right)=\rho\left(e_{1} \cdots e_{n}\right)
$$

and

$$
e_{0}^{-1} \rho\left(e_{0} \cdots e_{n-1}\right)=\rho\left(e_{1} \cdots e_{n-1}\right)
$$

Since

$$
I\left(e_{1} \cdots e_{n}\right) \subseteq I\left(e_{1} \cdots e_{n-1}\right)
$$

it is easy to see that this forces

$$
f^{-1} I\left(e_{1} \cdots e_{n}\right) \cap I\left(e_{0} \cdots e_{n-1}\right)=I\left(e_{0} \cdots e_{n}\right) .
$$

We argue similarly if $e_{0} \cdots e_{n}$ is an extreme right path following $0, e_{0} 0$.

(ii) Suppose $e_{0} \cdots e_{n-1} \in \Sigma_{F}$ but $e_{0} \cdots e_{n} \notin \Sigma_{F}$.

Using (i) repeatedly,

$$
f^{n}{ }_{\mid I\left(e_{0} \cdots e_{n-1}\right)}=e_{n-1}^{-1} \cdots e_{0}^{-1} .
$$

$f^{n}\left(\rho\left(e_{0} \cdots e_{n-1}\right)\right)$ is the endpoint of the extreme right path following $e_{n-1}^{-1} \cdots e_{0}^{-1} 0$, $e_{n-1}^{-1} \cdots e_{1}^{-1} 0, \ldots, 0$. Since $e_{0} \cdots e_{n} \notin \Sigma_{F}$, the edge $0, e_{n} 0$ lies either to the right of the extreme right path following $e_{n-1}^{-1} \cdots e_{0}^{-1} 0, \ldots, 0$ or to the left of the extreme 
left path. Suppose for definiteness we are in the first case. Then one sees that the points $\rho\left(e_{n}\right), \lambda\left(e_{n}\right), f^{n}\left(\rho\left(e_{0} \cdots e_{n-1}\right)\right), f^{n}\left(\lambda\left(e_{0} \cdots e_{n-1}\right)\right)$ occur in anticlockwise order round $S^{1}$ and the only possible coincidence of points is $\lambda\left(e_{n}\right)=f^{n}\left(\rho\left(e_{0} \cdots e_{n-1}\right)\right)$.

CoROLlaRY 4.9. (i) If $e_{0} \cdots e_{n} \in \Sigma_{F}$ then $\bigcap_{r=0}^{n} f^{-r} I\left(e_{r}\right)=I\left(e_{0} \cdots e_{n}\right)$.

(ii) If $e_{0} \cdots e_{n-1} \in \Sigma_{F}, e_{0} \cdots e_{n} \notin \Sigma_{F}$ then $\bigcap_{r=0}^{n} f^{-r} I\left(e_{r}\right)=\varnothing$ or is an endpoint of $I\left(e_{0} \cdots e_{n-1}\right)$.

Proof. (i) This follows easily from 4.8 (i) by an inductive argument.

(ii) Apply (i) to $I\left(e_{0} \cdots e_{n-1}\right)$ and then apply 4.8 (ii).

THEOREM 4.10. $\pi(\Sigma)=\Lambda=\bigcap_{r=0}^{\infty} f^{-r} Q$.

Proof. We have already shown

$$
\pi(\Sigma) \subseteq \Lambda \subseteq \bigcap_{r=0}^{\infty} f^{-r} Q
$$

Suppose $x \in \bigcap_{r=0}^{\infty} f^{-r} I\left(e_{r}\right)$. By corollary 4.9(ii), either $e_{0} \cdots e_{n} \in \Sigma_{F}$ for each $n$, or there is an $N$ so that $e_{0} \cdots e_{N-1} \in \Sigma_{F}, e_{0} \cdots e_{N} \notin \Sigma_{F}$ and $\bigcap_{r=0}^{N} f^{-r} I\left(e_{r}\right)=\{x\}$ is an endpoint of $I\left(e_{0} \cdots e_{N-1}\right)$. Since endpoints of such intervals are by definition endpoints of admissible paths in $G(\Gamma)$, and hence in $\pi(\Sigma)$, in the second case we have finished.

Otherwise, $e_{0} e_{1} \cdots \in \Sigma$. Let $y=\pi\left(e_{0} e_{1} \cdots\right)$. Then $y \in I\left(e_{0} e_{1} \cdots e_{n}\right)$ for all $n$ by 4.4 and $x \in I\left(e_{0} \cdots \dot{e}_{n}\right)$ by 4.9 . Since by $4.5,\left|I\left(e_{0} \cdots e_{n}\right)\right| \rightarrow 0$ as $n \rightarrow \infty, x=y$.

REMARK 4.11. The above shows that the f-expansions of a point in $\Lambda$ coincide with the representation as a point in $\pi(\Sigma)$, except perhaps at endpoints of intervals $I\left(e_{0} \cdots e_{n}\right)$. Further investigation would show these are exactly the points where $f$ is two-valued and where $\pi$ fails to be bijective, and in fact the two representations agree everywhere.

Let $W=\left\{\pi(x) \in \Lambda: x \in \Sigma, x=B_{1} B_{2} \cdots\right\}$, where $\left(B_{i}\right)_{i=1}^{\infty}$ is a sequence of consecutive left or right cycles, and where $B_{2} B_{3} \cdots$, is extreme. $W$ is finite and invariant under $\sigma$, hence $f(W) \subseteq W$, whichever value of $f$ we choose. (We include the case when $B_{2} B_{3} \cdots$ is the left or right boundary of an infinite sided polygon in $G(\Gamma)$, and the case $\left|B_{1}\right|=0$.)

THEOREM 4.12. $\Sigma$ is conjugate to a subshift of finite type, by a map which is bijective except at a countable number of points.

Proof. $W$ includes the endpoints of all the intervals $I(g), g \in \Gamma_{0}$, since these are extreme left or right paths. Therefore $W$ partitions $Q$ into a finite number of intervals $\mathscr{P}=\left\{I_{i}\right\}_{i=1}^{k}$. Since $f(W) \subseteq W$, we have $f\left(I_{i}\right) \cap I_{j}=\varnothing$ or $f\left(I_{i}\right) \supseteq I_{j}$, for all $i, j$. Let $\Delta=\left\{\left(i_{r}\right)_{r=0}^{\infty}: f\left(I_{i_{r}}\right) \supseteq I_{i_{r+1}}, r=0,1, \ldots\right\} . \Delta$ is a subshift of finite type [1]. Write $\psi(j)=e \in \Gamma_{0}$ if $I\left(i_{j}\right) \subseteq I(e)$. Define

$$
\psi: \Delta \rightarrow \Sigma \text { by } \psi\left(\left(i_{r}\right)\right)=\left(\psi\left(i_{r}\right)\right)
$$


This is possible since $\left(i_{r}\right) \in \Delta$ implies $\bigcap_{r=0}^{n} f^{-r} I_{i_{r}}$ contains more than one point for each $n$, so that by corollary $4.9, \psi\left(i_{0}\right) \cdots \psi\left(i_{n}\right) \in \Sigma_{F} . \psi$ fails to be bijective only at points where $\pi\left(\psi\left(i_{r}\right)\right) \in \Lambda$ has more than one $f$-expansion (relative to $Q$ ). But this is only a countable set.

Let $E=\{x \in \Lambda: \pi$ is not bijective at $x\}$.

THEOREM 4.13. f and $\Gamma$ are orbit equivalent on $\Lambda-E$; i.e., if $x, y \in \Lambda-E$ then $x=g y$, $g \in \Gamma$, if and only if $f^{n} x=f^{m} y$ for some $n, m \geq 0$.

Proof. From the definition of $f$ one has $f^{n} x=f^{m} y$ implies $x=g y, g \in \Gamma$. Since $\Gamma E \subseteq E$, it is enough to prove the converse for $g \in \Gamma_{0}$. If $x=\pi\left(\left(e_{i}\right)\right),\left(e_{i}\right) \in \Sigma$, and for some $n, g e_{0} \cdots e_{n}=f_{0} \cdots f_{m}$ where $f_{0} \cdots f_{m} e_{n+1} \cdots \in \Sigma$ then by lemma 4.3 one has $y=$ $g x=g \pi\left(\left(e_{i}\right)\right)=\pi\left(f_{0} \cdots f_{m} e_{n+1} \cdots\right)$ so that $f^{m}(y)=\pi\left(e_{n+1} e_{n+2} \cdots\right)=f^{n}(x)$.

Thus we only need investigate the cases in which the situation of lemma 4.3 fails. This means that $e_{0} \neq g^{-1}$, and that $g e_{0} \cdots e_{n}$ is excessive whenever $e_{0} \cdots e_{n}$ is extreme.

Suppose $e_{0} \cdots e_{n}$ is extreme and $g e_{0} \cdots e_{n}$ excessive. Then $g e_{0} \cdots e_{n}$ is the right or left boundary of a polygonal chain $L=\bigcup_{i=0}^{N} P_{i}$, and $g$ lies in the same polygon as $e_{0}$. Let $C=g^{-1} c_{0} \cdots c_{s}$ be the opposite boundary of $L$ to $e_{0} \cdots e_{n}$. Using the notation of lemma 3.3, one verifies by an easy inductive argument that $\partial L_{N-1}^{R}$, $\partial L_{N-1}^{L}$ are both extreme and

$$
\left|\partial L_{N-1}^{R}\right|-\left|\partial L_{N-1}^{L}\right|=\frac{1}{2}\left[\sigma\left(\partial L_{N-1}^{R}\right)-\tau\left(\partial L_{N-1}^{L}\right)\right] .
$$

Now taking all possible combinations in turn: $C$ the right or left boundary; $\left|\partial P_{N}\right|$ odd or even; $\left|\partial L_{N-1}^{R}\right|-\left|\partial L_{N-1}^{L}\right|=0$ or 1 ; one verifies that either $C e_{n+1} e_{n+2} \cdots \in \Sigma$ or that $g c_{0} \cdots c_{s-1} \in \Sigma_{F}, g^{-1} c_{0} \cdots c_{s} \notin \Sigma_{F}$ and $n=s$. For example, suppose that $C$ is the right boundary and $\left|\partial L_{N-1}^{R}\right|-\left|\partial L_{N-1}^{L}\right|=0$. Then if $\left|\partial P_{N}\right|$ is even, $\left|\partial P_{N}^{L}\right|=$ $\frac{1}{2}\left(\left|\partial P_{N}\right|-2\right)$, so $\left|\partial P_{N}^{R}\right|=\frac{1}{2}\left|\partial P_{N}\right|$, and $C e_{n+1} \cdots$ is admissible because $c_{s} \neq e_{n+1}^{-1}$ or $e_{0} \cdots e_{n+1}$ would be excessive, and $C e_{n+1}$ is not excessive because $e_{n+1} \neq e_{n}^{-1}$. If $\left|\partial P_{N}\right|$ is odd, $\left|\partial P_{N}^{L}\right|=\frac{1}{2}\left(\left|\partial P_{N}\right|-3\right)$ so $\left|\partial P_{N}^{R}\right|=\frac{1}{2}\left(\left|\partial P_{N}\right|+1\right)$. Then $n=s, g^{-1} c_{0} \cdots c_{n-1}$ is extreme and $g^{-1} c_{0} \cdots c_{n}$ is not admissible.

In the first case $\left(C e_{n+1} \cdots \in \Sigma\right)$, we are in the situation of lemma 4.3. Otherwise consider $e_{n} e_{n+1} \cdots \in \Sigma$ and apply $c_{n}$. Either there are $m_{1}, m_{2}$ so that $f^{m_{1}}\left(e_{n} e_{n+1} \cdots\right)=f^{m_{2}}\left(c_{n} e_{n+1} \cdots\right)$, in which case, since $g e_{0} \cdots e_{n}=c_{0} \cdots c_{n}$,

$$
\begin{aligned}
f^{n+m_{2}}\left(g e_{0} \cdots e_{n} e_{n+1} \cdots\right) & =f^{n+m_{2}}\left(c_{0} \cdots c_{n} e_{n+1} \cdots\right) \\
& =f^{m_{2}}\left(c_{n} e_{n+1} \cdots\right) \\
& =f^{m_{1}}\left(e_{n} e_{n+1} \cdots\right) \\
& =f^{n+m_{1}}\left(e_{0} \cdots e_{n} e_{n+1} \cdots\right)
\end{aligned}
$$

or we have $n_{1}$ and $c_{0}^{\prime} \cdots c_{n_{1}}^{\prime}$ so that

$$
c_{n} e_{n} e_{n+1} \cdots e_{n_{1}}=c_{0}^{\prime} \cdots c_{n_{1}}^{\prime}, c_{n}^{-1} c_{0}^{\prime} \cdots c_{n_{1}-1}^{\prime} \in \Sigma_{F}, c_{n}^{-1} c_{0}^{\prime} \cdots c_{n_{1}}^{\prime} \notin \Sigma_{F} .
$$

On repeating this argument, either we eventually find $f^{k}\left(g e_{0} \cdots\right)=f^{l}\left(e_{0} \cdots\right)$ for some $k, l$, or we see that for infinitely many $n, e_{0} e_{1} \cdots e_{n}$ is the left (or right) 
boundary of a chain in $G(\Gamma)$ whose opposite boundary is also admissible, so that $e_{0} \cdots e_{n} \cdots$ lies in $E$.

Remark 4.14. The above proof also shows how to describe the action of $\Gamma$ on $\Delta$ as an action on $\Sigma$. The effect of certain $g \in \Gamma_{0}$ on points in $E$ is to 'flip' an extreme left path into an extreme right path, and vice versa.

\section{The critical exponent $\delta$}

In this section we shall restrict our attention to groups $\Gamma$ with no parabolic elements (and of course with non-exceptional graphs). We show the existence of a number $\delta$ with the properties listed in the introduction. We keep the notation of previous sections. We shall work mainly on the sequence space $\Delta$. Let $\Delta_{F}=$ $\left\{i_{0} \cdots i_{n}: \bigcap_{r=0}^{n} f^{-r} I\left(i_{r}\right)\right.$ is an interval $\}$. The corresponding cylinder sets in $\Delta$ we denote $Z\left(i_{0} \cdots i_{n}\right)$.

Let

$$
I\left(i_{0} \cdots i_{n}\right)=\bigcap_{r=0}^{n} f^{-r} I\left(i_{r}\right), \quad I\left(i_{r}\right) \in \mathscr{P} .
$$

THEOREM 5.1. There exist $N \in \mathbb{N}$ and $\beta>1$ so that $\left(f^{N}\right)^{\prime}(x)>\beta$ for all $x \in \bigcap_{r=0}^{N} f^{-r} Q$. Proof. Pick $i_{0} \cdots i_{n} \in \Delta_{F}$. Let

$$
\left.F=f_{\mid I\left(i_{0}\right.}^{n} \cdots i_{n}\right)=\psi\left(i_{n-1}\right)^{-1} \cdots \psi\left(i_{0}\right)^{-1} .
$$

Let

$$
k=\sup _{x, y \in Q}\left|f^{\prime \prime}(x) / f^{\prime}(y)\right|
$$

For $x, y \in I\left(i_{0} \cdots i_{n}\right)$,

$$
\begin{aligned}
\left|1-\frac{f^{\prime}\left(f^{r}(x)\right)}{f^{\prime}\left(f^{r}(y)\right)}\right| & =\left|\frac{f^{\prime \prime}(\xi)\left(f^{r}(x)-f^{r}(y)\right)}{f^{\prime}\left(f^{r}(y)\right)}\right| \quad \text { some } \xi \in I\left(i_{r} \cdots i_{n}\right) \\
& \leq k\left|I\left(i_{r} \cdots i_{n}\right)\right|
\end{aligned}
$$

By proposition 4.5,

$$
\left|I\left(i_{r} \cdots i_{n}\right)\right| \leq \gamma \exp (-\alpha(n-r)) .
$$

Hence $\sum_{r=0}^{n}\left|1-f^{\prime}\left(f^{r}(x)\right) / f^{\prime}\left(f^{r}(y)\right)\right|$ is bounded independent of $x, y$ or $n$ and therefore so is $\prod_{r=0}^{n}\left|f^{\prime}\left(f^{r} x\right) / f^{\prime}\left(f^{r} y\right)\right|$. Therefore

$$
\left|F^{\prime}(x) / F^{\prime}(y)\right| \leq c_{1}, \quad x, y \in I\left(i_{0} \cdots i_{n}\right) .
$$

Since $F: I\left(i_{0} \cdots i_{n}\right) \rightarrow I\left(i_{n}\right)$ is a bijection,

Using (5.1.1),

$$
\int_{I\left(i_{0} \cdots i_{n}\right)} F^{\prime}(y) d y=\left|I\left(i_{n}\right)\right| .
$$

$$
c^{-1} M\left(i_{0} \cdots i_{n}\right)^{-1} \leq\left|I\left(i_{0} \cdots i_{n}\right)\right| \leq c M\left(i_{0} \cdots i_{n}\right)^{-1} \text { for some } c>0,
$$


where

$$
M\left(i_{0} \cdots i_{n}\right)=\sup \left\{\left|F^{\prime}(x)\right|: x \in I\left(i_{0} \cdots i_{n}\right)\right\}
$$

and using $\left|I\left(i_{0} \cdots i_{n}\right)\right| \leq \gamma \exp (-\alpha n)$,

$$
\left|F^{\prime}(x)\right| \geq c_{2} \exp (\alpha n), \quad x \in I\left(i_{0} \cdots i_{n}\right) .
$$

In particular, choosing $c_{2} \exp (\alpha N)>1$ gives the result.

Remark 5.2. We shall later need to modify this result and replace the intervals $I\left(i_{0} \cdots i_{n}\right)$ by slightly larger intervals $J\left(i_{0} \cdots i_{n}\right)$, as follows. Given $J(i) \supset I(i)$, $I(i) \in \mathscr{P}$, a family of open neighbourhoods of $I(i)$, set $J\left(i_{0} \cdots i_{n}\right)=F^{-1} J\left(i_{0}\right)$ where $F=\psi\left(i_{n}\right)^{-1} \cdots \psi\left(i_{0}\right)^{-1}$ is an extension of the $F$ in 5.1. Choose the $J(i)$ small enough that

We deduce that

$$
\left|\left(f^{N}\right)^{\prime}(x)\right| \geq \beta^{\prime}>1 \quad \text { for all } x \in J\left(i_{0} \cdots i_{N}\right)
$$

$$
\left|J\left(i_{0} \cdots i_{n}\right)\right| \leq k_{1} \lambda^{n}
$$

for a constant $k_{1}$, some $\lambda<1$ and all sufficiently large $n$. The proof of 5.1 then shows that (5.1.1), (5.1.2), (5.1.3) all hold with different choice of constants and $J$ replacing $I$.

COROLlARY 5.3. There exists a constant $\alpha>0$ such that $B_{\delta}(x) \subseteq J\left(i_{0} \cdots i_{n}\right)$ for any $x \in I\left(i_{0} \cdots i_{n}\right)$ and $i_{0} \cdots i_{n} \in \Delta_{F}$, where $F=\psi\left(i_{n}\right)^{-1} \cdots \psi\left(i_{0}\right)^{-1}$ as above, and $\delta=\alpha\left|F^{\prime}(x)\right|^{-1}$.

Proof. Find $\varepsilon>0$ so that $B_{\varepsilon}(y) \subseteq J\left(i_{0}\right)$ whenever $y \in I\left(i_{0}\right) \in \mathscr{P} . \quad B_{\eta}\left(F^{-1}(y)\right) \subset$ $F^{-1} J\left(i_{0}\right)=J\left(i_{0} \cdots i_{n}\right)$ whenever $\eta \sup \left\{\left|F^{\prime}(y)\right|: y \in J\left(i_{0} \cdots i_{n}\right)\right\}<\varepsilon$. Using (5.1.1) gives the result.

Define $\phi: \Delta \rightarrow \mathbb{R}$ by

$$
\phi\left(\left(i_{r}\right)\right)=-\log \mid\left(\psi\left(i_{0}\right)^{-1}\right)^{\prime}\left(\pi\left(\left(i_{r}\right)\right) \mid\right.
$$

LEMMA 5.4. $\phi$ is Hölder continuous on $\Delta$, i.e. there are constants $d>0, \nu<1$ so that $|\phi(x)-\phi(y)| \leq d \nu^{n+1}$ whenever $x_{i}=y_{i}, i=0, \ldots, n$.

Proof.

$$
\begin{aligned}
|\log | f^{\prime}(x)|-\log | f^{\prime}(y) \| & =\int_{\left|f^{\prime}(x)\right|}^{\left|f^{\prime}(y)\right|} \frac{d t}{t} \\
& \leq\left|f^{\prime}(y)-f^{\prime}(x)\right| \inf _{x \in Q} \frac{1}{\left|f^{\prime}(x)\right|} \\
& \leq|y-x| \sup _{x, \xi \in Q}\left|\frac{f^{\prime \prime}(\xi)}{f^{\prime}(x)}\right| \\
& \leq k\left|I\left(x_{0} \cdots x_{n}\right)\right| .
\end{aligned}
$$

Now use the proof of 4.5 . 
We can now apply the theory of Gibbs states $[1]$ on $\Delta$ to define $\delta$ and $\mu$ (see introduction).

Let $S_{n} \phi(x)=\sum_{r=0}^{n-1} \phi\left(\sigma^{r} x\right)$ and note that for $x \in Z\left(i_{0} \cdots i_{n-1}\right)$,

$$
S_{n} \phi(x)=-\log \left|\prod_{r=0}^{n-1} f^{\prime}\left(f^{r}(x)\right)\right|=-\log \left|\left(f^{n}\right)^{\prime}(x)\right| .
$$

For $s \geq 0$, let

$$
Z_{n}(s \phi)=\sum_{i_{0} \cdots i_{n-1} \in \Delta_{F}} m\left(i_{0} \cdots i_{n-1}\right)^{-s},
$$

where $m\left(i_{0} \cdots i_{n-1}\right)=\inf \left\{\left|f^{n^{\prime}}(x)\right|: x \in Z\left(i_{0} \cdots i_{n-1}\right)\right\}$. By [1] p. 30,

$$
P(s \phi)=\lim _{n \rightarrow \infty}(1 / n) \log Z_{n}(s \phi)
$$

exists and is called the pressure of $s \phi . P(s \phi)$ is a decreasing function of $s$ and $\sum_{n=0}^{\infty} Z_{n}(s \phi)$ converges if $P(s \phi)>0$, diverges if $P(s \phi)<0 . \sum Z_{n}(s \phi)$ is a Dirichlet series, so there must be a unique $\delta$ so that $P(\delta \phi)=0$.

According to [1] there is a probability measure $\mu$ on $\Delta$ such that:

There are constants $K_{1}, K_{2}$ so that

$$
K_{1} \leq \frac{\mu\left(Z\left(i_{0} \cdots i_{n-1}\right)\right)}{\exp \left(\delta S_{n} \phi(x)\right)} \leq K_{2}
$$

whenever $i_{0} \cdots i_{n-1} \in \Delta_{F}$ and $x \in Z\left(i_{0} \cdots i_{n-1}\right)$.

$\mu$ is invariant and ergodic for $\sigma$.

There is a positive Hölder continuous function $h$ on $\Delta$, and a probability measure $\nu$, so that $\mu=h \nu$ and $\mathscr{L} h=h, \mathscr{L}^{*} \nu=\nu$, where $\mathscr{L}$ is the operator

$$
\mathscr{L} f(x)=\sum_{y \in \sigma^{-1} x} \exp (\phi(y)) f(y),
$$

$f$ a function on $\Delta$, and $\mathscr{L}^{*}$ the dual of $\mathscr{L}$. Notice that (5.4.1) and (5.4.3) use $P(\delta \phi)=0$.

Applying (5.4.3) to the characteristic function of $Z\left(i_{0}\right)$, where $a_{0}=\psi\left(i_{0}\right)$, we obtain

$$
\frac{d a_{0 *} \nu}{d \nu}(x)=\left|a_{0}^{\prime}(x)\right|^{\delta} \quad \text { whenever } x \in f\left(I\left(a_{0}\right)\right) \text {. }
$$

Applying $F=f^{n}$ to $Z\left(i_{0} \cdots i_{n-1}\right)$ and using (5.4.1) and (5.1.1) we obtain constants $K_{1}^{\prime}, K_{2}^{\prime}$ so that

$$
K_{1}^{\prime} \leq \frac{\mu\left(Z\left(i_{0} \cdots i_{n-1}\right)\right)}{\left|I\left(i_{0} \cdots i_{n-1}\right)\right|^{\delta}} \leq K_{2}^{\prime} .
$$

THEOREM 5.5. The Hausdorff dimension of $\Lambda$ is $\delta$. The $\delta$-dimensional Hausdorff measure of $\Lambda$ is finite and equivalent to $\mu$.

Proof. The proof is essentially that of lemma 10 of [2]. One has only to read $J\left(i_{0} \cdots i_{n}\right)$ for $D\left(x_{0} \cdots x_{n}\right)$ and $\Lambda$ for $\gamma$, and replace lemma 9 by corollary 5.3.

PROpOsition 5.6. For $g \in \Gamma$ and $x \in \Lambda,\left(d g_{*} \nu / d \nu\right)(x)=\left|g^{\prime}(x)\right|^{\delta} . \mu$ (and hence $\nu$ ) is ergodic for $\Gamma$. 
Proof. Let $x=a_{0} a_{1} \cdots, g x=b_{0} b_{1} \cdots$ be the $f$-expansions of $x, g x$ relative to $Q$. By theorem 4.12 except for a countable number of $x$, there exist $n, m$ so that $a_{n+r}=b_{m+n} r \geq 0$, and $g=b_{0} \cdots b_{m} a_{n}^{-1} \cdots a_{0}^{-1}=e_{n+m+1} \cdots e_{0}$, say.

By (5.4.4) we have

$$
\frac{d a_{*} \nu}{d \nu}(y)=\left|a^{\prime}(y)\right|^{\delta} \quad \text { for } \quad a \in \Gamma_{0}, y \in \Lambda,
$$

whenever $y=c_{0} c_{1} \cdots \in \Sigma$ is such that $a c_{0} c_{1} \cdots \in \Sigma$. Thus

$$
\frac{d g_{*} \nu}{d \nu}(x)=\prod_{r=0}^{n+m+1}\left|e_{r}^{\prime}\left(e_{r-1} \cdots e_{0} x\right)\right|^{\delta}=\left|g^{\prime}(x)\right|^{\delta} .
$$

Ergodicity follows from (5.4.2) and theorem 4.13.

Corollary 5.7. $0<\delta \leq 1$, and $\delta=1$ if and only if $\Lambda=S^{1}$ and $D / \Gamma$ is compact.

Proof. Since $\delta=$ Hausdorff $\operatorname{dim}(\Lambda), 0 \leq \delta \leq 1$.

If $\delta=0, \nu$ is $\Gamma$-invariant. But it is easy to see that this is impossible unless $\Gamma$ is an elementary group. (If $\nu$ is invariant for a hyperbolic element then all the mass of $\nu$ is concentrated at the fixed points.)

If $\delta=1$, then $\nu$ is equivalent to Lebesgue measure, so $\Lambda=S^{1}$. Conversely, if $\Lambda=S^{1}$, Hausdorff $\operatorname{dim}(\Lambda)=1=\delta . D / \Gamma$ is compact since we are assuming that $\Gamma$ has no cusps.

COROLlaRY 5.8. $\nu$ is $\delta$-dimensional Hausdorff measure $h_{\delta}$ on $\Lambda$.

Proof. By 5.7, $h_{\delta}$ is equivalent to $\nu$. It is clear that $h_{\delta}$ transforms according to the same law as $\nu$ (look at the change in measure for small balls near $x$ ). Hence $d h_{\delta} / d \nu$ is a $\Gamma$-invariant function on $\Lambda$. Ergodicity of $\Gamma$ with respect to $\nu$ gives the result.

Corollary 5.9. $\nu$ is the so-called Patterson measure constructed in [8] and [9]. Proof. [9] theorems 7 and 8, and [8] theorem 7.2.

It follows from [9] that $\delta$ is the exponent of convergence of $\Gamma$. We shall prove this directly from our constructions.

LemMA 5.10. The Dirichlet series

$$
\sum_{i_{0} \cdots i_{n} \in \Delta_{F}}\left|I\left(i_{0} \cdots i_{n}\right)\right|^{-s} \text { and } \sum_{a_{0} \cdots a_{n} \in \Sigma_{F}}\left|I\left(a_{0} \cdots a_{n}\right)\right|^{-s}
$$

have the same exponent of convergence.

Proof. We have

$$
I\left(a_{0} \cdots a_{n}\right)=\bigcup\left\{I\left(i_{0} \cdots i_{n}\right): \psi\left(i_{r}\right)=a_{n} 0 \leq r \leq n\right\} .
$$

Let $\rho(n)$ be the number of sets in this union. It is clear that the argument of theorem 5.1 applied to $I\left(a_{0} \cdots a_{n}\right)$ will give

$$
\frac{1}{c^{\prime}} M^{\prime}\left(a_{0} \cdots a_{n}\right)^{-1} \leq\left|I\left(a_{0} \cdots a_{n}\right)\right| \leq c^{\prime} M^{\prime}\left(a_{0} \cdots a_{n}\right)^{-1},
$$


where $M^{\prime}\left(a_{0} \cdots a_{n}\right)=\sup \left\{F^{\prime}(x): x \in I\left(a_{0} \cdots a_{n}\right)\right\}$. Using (5.1.1) applied to $I\left(a_{0} \cdots a_{n}\right) \supseteq I\left(i_{0} \cdots i_{n}\right)$, one obtains a constant $c^{\prime \prime}$ so that

$$
c^{\prime \prime} \leq \frac{\left|I\left(i_{0} \cdots i_{n}\right)\right|}{\left|I\left(a_{0} \cdots a_{n}\right)\right|} \leq c^{\prime \prime}
$$

whenever $I\left(i_{0} \cdots i_{n}\right) \subseteq I\left(a_{0} \cdots a_{n}\right)$.

Hence $\rho(n) \leq$ const. independent of $n$. Now

$$
\begin{aligned}
\sum_{i_{0} \cdots i_{n} \in \Delta_{F}}\left|I\left(i_{0} \cdots i_{n}\right)\right|^{-s} & =\sum_{a_{0} \cdots a_{n} \in \Sigma_{F}}\left(\sum_{I\left(i_{0} \cdots i_{n}\right) \subseteq I\left(a_{0} \cdots a_{n}\right)}\left|I\left(i_{0} \cdots i_{n}\right)\right|^{-s}\right) \\
& \leq c^{\prime \prime} \sum_{a_{0} \cdots a_{n} \in \Sigma_{F}} \rho(n)\left|I\left(a_{0} \cdots a_{n}\right)\right|^{-s} \\
& \leq \text { const. } \sum_{a_{0} \cdots a_{n} \in \Sigma_{F}}\left|I\left(a_{0} \cdots a_{n}\right)\right|^{-s} .
\end{aligned}
$$

The reverse inequality is similar.

LEMMA 5.11. There is a constant $d>0$ so that

$$
d^{-1} \leq \frac{\left|I\left(a_{0} \cdots a_{n}\right)\right|}{\log H\left(0, a_{0} \cdots a_{n} 0\right)} \leq d
$$

whenever $a_{0} \cdots a_{n} \in \Sigma_{F}$.

Proof. Let $\alpha$ be the angle between the non-Euclidean lines joining $a_{0} \cdots a_{n} 0$ to $\lambda\left(a_{0} \cdots a_{n}\right)$ and $\rho\left(a_{0} \cdots a_{n}\right)$. We have $0<\alpha_{0} \leq \alpha \leq \alpha_{1}<\pi$ independently of $n$ (apply the transformation $a_{n}^{-1} \cdots a_{0}^{-1}$ and consider the corresponding paths starting at 0$)$. Let $H\left(0, a_{0} \cdots a_{n} 0\right)=r$. Rotate the disk so that the points $\lambda\left(a_{0} \cdots a_{n}\right)$, $\rho\left(a_{0} \cdots a_{n}\right)$ are symmetrically placed with respect to the real axis and then apply the transformation

$$
T=\left(\begin{array}{rr}
\cosh \frac{1}{2} r & -\sinh \frac{1}{2} r \\
-\sinh \frac{1}{2} r & \cosh \frac{1}{2} r
\end{array}\right) .
$$

This carries the non-Euclidean rays $a_{0} \cdots a_{n} 0, \lambda\left(a_{0} \cdots a_{n}\right)$ and $a_{0} \cdots a_{n} 0$, $\rho\left(a_{0} \cdots a_{n}\right)$ to radii through 0 at an angle $\alpha$. One computes easily that there is a constant $d^{\prime}$ so that

$$
d^{\prime-1} \exp r \leq\left|T^{\prime}(\exp (i \theta))\right| \leq d^{\prime} \exp r
$$

for all sufficiently large $r$ and $\theta \leq \alpha_{1}$. This gives the result.

Corollary 5.12. The Poincaré series $\sum_{g \in \Gamma} \exp (-s H(0, g 0))$ and the Dirichlet series $\sum_{i_{0} \cdots i_{n} \in \Delta_{F}}\left|I\left(i_{0} \cdots i_{n}\right)\right|^{-s}$ have the same exponent of convergence.

Proof. This follows immediately from lemmas 5.10, 5.11 and the fact that $a_{0} \cdots a_{n} \in \Sigma_{F}$ runs exactly once over all elements of $\Gamma$.

Corollary 5.13. The Poincaré series $\sum_{g \in \Gamma} \exp (-s H(0, g 0))$ diverges at $s=\delta$.

Proof. By lemma 5.11, (5.10.1) and (5.4.5) $\sum_{|g|=n} \exp (-\delta H(0, g 0))$ is comparable to

$$
\sum_{i_{0}} \mu\left(I\left(i_{0} \cdots i_{n}\right)\right)=1
$$




\section{REFERENCES}

[1] R. Bowen. Equilibrium States and the Ergodic Theory of Anosov Diffeomorphisms. Lecture Notes in Math. no. 470. Springer: Berlin, 1975.

[2] R. Bowen. Hausdorff dimension of quasi-circles. Publ. Math. IHES 50 (1979), 1-25.

[3] R. Bowen \& C. Series. Markov maps associated with Fuchsian groups. Publ. Math. IHES 50 (1979), 153-179.

[4] M. Dehn. Transformation der Kurven auf zweiseitigen Flächen. Math. Ann. 72 (1912), 413-421.

[5] W. Floyd. Group completions and limit sets of Kleinian groups. Inventiones Math. 57 (1980), 205-218.

[6] L. R. Ford. Automorphic Functions. McGraw Hill: New York, 1929.

[7] B. Maskit. On Poincaré's theorem for fundamental polygons. Adv. Math. 7 (1971), 219-230.

[8] S. J. Patterson. The limit set of a Fuchsian group. Acta Math. 136 (1976), 241-273.

[9] D. Sullivan. The density at infinity of a discrete group of hyperbolic motions. Publ. Math. IHES 50 (1979), 171-202. 